\title{
Análisis del Desempeño de Estudiantes en Tareas Matemáticas. Estudio Exploratorio en el Instituto Politécnico Nacional de México
}

\author{
Martha L. García ${ }^{1}$, Alicia López ${ }^{2}$ y Alejandra Díaz ${ }^{2}$ \\ 1 Escuela Superior de Ingeniería Mecánica y Eléctrica, Dpto. de Comunicaciones y Electrónica, Instituto \\ Politécnico Nacional, Ciudad de México-México (e-mail: mlgarcia@ipn.mx) \\ ${ }^{2}$ Facultad de Matemáticas Aplicadas, Universidad Juárez del Estado de Durango, Durango-México \\ (e-mail: ablopez@ujed.mx; alejandradiazsoto01@gmail.com)
}

Recibido Nov. 22, 2017; Aceptado Ene. 22, 2018; Versión final Abr. 11, 2018, Publicado Oct. 2018

\begin{abstract}
Resumen
El objetivo del estudio de tipo exploratorio descriptivo, fue determinar el desempeño de los estudiantes en tareas matemáticas con diferentes niveles de demanda cognitiva. Se seleccionaron y clasificaron un conjunto de tareas en siete áreas: álgebra, geometría, aritmética, manejo de información, geometría analítica, y cálculo diferencial e integral. Participaron 225 estudiantes de un total de 510 que ingresaron a una de las 23 carreras de ingeniería que se ofertan en el Instituto Politécnico Nacional en México. Se determinó el número de estudiantes que resolvieron con éxito las tareas en cada uno de los niveles, así como las características de las tareas que presentaron mayor dificultad. Los resultados permiten identificar los temas en los que los estudiantes se desempeñan mejor. También aportan evidencia de que los estudiantes pueden tener un mejor desempeño en tareas orientadas a un concepto aun cuando no puedan aplicar con éxito reglas y procedimientos sobre el mismo.
\end{abstract}

\section{Analysis of Performance of University Students in Math Problems. Exploratory Study in the National Polytechnic Institute of Mexico}

\begin{abstract}
This research presents the results of a descriptive exploratory study to determine the students' performance in solving mathematical problems with different levels of cognitive demand. A set of tasks was selected and classified into seven areas: algebra, geometry, arithmetic, information management, analytical geometry, differential and integral calculus. A sample of 225 students from a total of 510 that entered any of the 23 engineering courses offered at the National Polytechnic Institute in Mexico. The number of students who successfully solved the tasks in each of the levels was determined, as well as the characteristics of the tasks that presented the greatest difficulty. The results of the research allow identifying the subjects in which students perform better. They also provide evidence that students can perform better in concept-oriented tasks even when they cannot successfully apply rules and procedures about it.
\end{abstract}

Keywords: cognitive demand, test, knowledge, mathematical task 


\section{INTRODUCCIÓN}

Existen diferentes investigaciones en las que se analiza el desempeño que muestran los estudiantes cuando cambian de un nivel educativo a otro. En el campo de la matemática los resultados no son alentadores, en el reporte presentado por el grupo de estudio cuatro del ICME 12 se señala que en los últimos años se ha identificado una aparente disminución en habilidades técnicas, poder de análisis y percepción de la importancia que tiene la precisión y demostración en matemáticas, en los estudiantes que ingresan a las universidades. Lo anterior parece estar relacionado con el éxito o fracaso que tienen en las matemáticas universitarias (Thomas et al., 2012). Estos resultados son reforzados con lo que se incluye en el informe español de Programa para la Evaluación Internacional de los Alumnos (MECD, 2016) en el que se evalúa además del conocimiento de los alumnos su capacidad para aplicar ese conocimiento a la vida real, una vez que han concluido la educación obligatoria. Los resultados muestran que de los países miembros de la Organización para la Cooperación y Desarrollo Económico (OCDE), el 23,4\% de los alumnos de 15 años se encuentra en los niveles $<1$ y 1 de desempeño en matemáticas; y que las puntuaciones más bajas se tienen en los estudiantes de Brasil y México. Estos jóvenes responden con éxito preguntas relacionadas con contextos matemáticos que les son familiares, en las que la información está claramente definida; son capaces de llevar a cabo procedimientos rutinarios y seguir instrucciones directas.

En la misma línea, es posible encontrar investigaciones sobre la falta de fluidez y habilidad en técnicas básicas de matemáticas y de fundamentos para desarrollar las matemáticas universitarias. Varsavsky (2010) asocia la débil preparación con que ingresan los estudiantes a la educación superior con la disminución del número de graduados con fuertes habilidades en matemáticas. Armstrong, y Croft, (1999) concluyen, a partir de los resultados de un examen de opción múltiple aplicado a 563 estudiantes al inicio del primer año escolar, que las universidades deben adaptar sus cursos para contrarrestar el déficit que los aspirantes para cursar una carrera de ingeniería presentan, en habilidades matemáticas básicas. Etxeberria et al. (2017) obtuvieron información de la percepción que tienen los estudiantes sobre su nivel de conocimientos previos, antes y después de cursar la asignatura de Álgebra y Geometría en el primer cuatrimestre de una carrera de ingeniería en Bilbao. Los autores reportan que el nivel de formación previa que creen tener los estudiantes es acorde con el contenido de la asignatura, destacan que las principales dificultades que los alumnos reconocieron al finalizar la asignatura fueron la falta de destreza en el manejo del cálculo básico y en su capacidad de razonamiento. Señalan también, que los resultados de la investigación son de primordial importancia para el diseño de contenidos teóricos y actividades prácticas que los alumnos de nuevo ingreso podrán utilizar antes de iniciar la asignatura.

En México, el Instituto Nacional para la Evaluación de la Educación (INEE) realizó en 2011 un diagnóstico de los conocimientos y habilidades en matemáticas de los estudiantes de educación media superior. Se encontró que el $30 \%$ de los estudiantes pueden reconocer la información y realizar procedimientos rutinarios siguiendo instrucciones precisas en situaciones explícitas; otro 30\% de los estudiantes pueden interpretar y reconocer situaciones en contextos que sólo requieren de una inferencia directa, utilizan algoritmos, fórmulas convencionales o procedimientos elementales y efectúan razonamientos directos; el $25 \%$ de los estudiantes ejecutan procedimientos que requieren decisiones secuenciales, seleccionan y aplican estrategias de resolución de problemas sencillos y pueden exponer mediante escritos breves, sus interpretaciones, resultados y razonamientos. Sólo el $15 \%$ de los estudiantes pueden trabajar con modelos explícitos en situaciones complejas y concretas, así como elaborar y comunican explicaciones y argumentos basados en sus interpretaciones y acciones (INEE, 2011).

En 2015 el INEE en coordinación con la Secretaría de Educación Pública, elaboraron realizaron un proyecto denominado Plan Nacional de Evaluación de los Aprendizajes (Planea), para determinar a nivel nacional los conocimientos y habilidades en matemáticas de los alumnos de bachillerato. Utilizaron un instrumento que incluyó 110 ítems que evalúan cuatro niveles de dominio en matemáticas (SEP, 2015). El INEE reportó que en la prueba Planea, el $51.3 \%$ de los estudiantes que participaron se ubican en el nivel de dominio I, cuentan con habilidad para identificar elementos de información que se incluyen de manera explícita en textos apelativos, argumentativos, expositivos y narrativos, estos resultados coinciden con los reportados en el informe español MECD (2016) para el caso de México.

Además de las investigaciones en las que se evalúan las destrezas y conocimientos matemáticos de los estudiantes, se identificaron investigación sobre lo que piensan los profesores sobre este problema, en el reporte del grupo de estudio cuatro del ICME 12, se reportan los resultados de una encuesta realizada a un grupo de 79 profesores de matemáticas universitarias de 21 países. Aproximadamente el $30 \%$ piensan que los estudiantes presentan problemas para transitar del bachillerato a la universidad debido a la falta de preparación en el bachillerato y de desarrollo de las habilidades básicas en matemáticas. En el reporte se menciona que de acuerdo con los profesores, un objetivo del bachillerato es que los estudiantes logren una profunda comprensión de las matemáticas. Sin embargo, aun cuando los profesores parecen estar de acuerdo 
con esta recomendación, hay grandes diferencias de lo que esto significa para ellos. Stanley y Sundström, (2007) afirman que una comprensión profunda se logra cuando se busca más allá de las características superficiales de una tarea y se reconoce la estructura matemática que subyace en ella. Otra vertiente se identifica en el trabajo de Garbin (2005) quién distingue entre pensamiento matemático elemental y avanzado, para pasar de uno a otro señala, existe una etapa de transición. En un primer momento se traspasa el aprendizaje del profesor al alumno, se incrementar en frecuencia y relevancia la demostración y la definición, y se favorecen los cambios del alumno sobre la manera de realizar sus tareas de rutina, cambia la forma en que se trata la información y se realizan los procesos matemáticos.

Los trabajos referenciados en los párrafos anteriores aportan información sobre la aparente disminución en la preparación y desarrollo de habilidades básicas en matemáticas en los estudiantes al ingresar a la universidad y sobre la importancia de que las instituciones universitarias determinen ¿Qué tipo de tareas son capaces de resolver con éxito? ¿En qué tareas se presentan las mayores dificultades? ¿Qué conocimiento ponen en juego para resolverlas? con el propósito de que puedan tomar acciones que contribuyan para que los estudiantes logren los conocimientos y habilidades que requieren para graduarse y desempeñarse con éxito en su vida profesional. Thomas et al. (2012) señalan, que existen diferentes aspectos relacionados con los problemas que se presentan en la transición del bachillerato a la universidad, que son de interés para investigadores y profesionales de la enseñanza; para abordar estas interrogantes se requiere un marco teórico que permita determinar en qué medida las puntuaciones obtenidas en una prueba reflejan ciertas formas de pensamiento y procesos cognitivos de niveles superiores asociados con una comprensión de las matemáticas, así como concepciones erróneas que se evidencien, y a partir de esta información proponer acciones para el mejorar el aprendizaje (Artavia, 2011).

Un modelo que se ha desarrollado para conocer el tipo y nivel de razonamiento que un estudiante requiere para comprometerse y resolver con éxito una tarea, acuñado como demanda cognitiva de una tarea, fue desarrollado por Stein et al. (2009). Los autores proponen una taxonomía con cuatro niveles para clasificar el tipo y nivel de razonamiento que una tarea demanda del estudiante: a) Bajo nivel de demanda cognitiva, en el que se privilegia la memorización; b) Bajo nivel de demanda cognitiva, con énfasis en los procedimientos, pero sin relación entre ellos; c) Alto nivel de demanda cognitiva en el que los estudiantes relacionan los procedimientos utilizados $y, d$ ) Alto nivel de demanda cognitiva, el trabajo se orienta al uso de teoremas y demostraciones de los mismos. La importancia de conocer la demanda cognitiva de una tarea radica en que propiciar oportunidades para favorecer el aprendizaje de un estudiante, va más allá de formar grupos de estudiantes para trabajar en forma colaborativa, o incorporar una calculadora durante la ejecución de una tarea; es el nivel y el tipo de pensamiento que la tarea demanda en el estudiante lo que determina su aprendizaje (Gaylord, 1991). El marco teórico desarrollado por Stein et al. (2009) describe un método para analizar una tarea, tal y como es presentada en forma impresa a un estudiante y para analizar la evolución de la tarea durante una clase; en la investigación que aquí se reporta el marco teórico se utiliza en el primer sentido.

La investigación tuvo como objetivo, determinar el desempeño de los estudiantes en tareas matemáticas con diferentes niveles de demanda cognitiva. Para lograr este objetivo, se seleccionaron y clasificaron un conjunto de tareas matemáticas en tres de los niveles de demanda cognitiva propuestos por Stein et al. (2009). Se determinó el número de estudiantes que resolvieron con éxito las tareas en cada uno de los niveles, así como las tareas que presentaron mayor dificultad, a partir del número de estudiantes que no pudieron dar una respuesta correcta. En la investigación participaron 225 estudiantes de un total de 510 que ingresaron a una de las 23 carreras de ingeniería que se ofertan en el Instituto Politécnico Nacional (IPN) en México.

\section{DISEÑO DE LA INVESTIGACIÓN, MÉTODOS Y PROCEDIMIENTOS}

La investigación se desarrolló en cuatro etapas: 1) se llevó a cabo la revisión de diferentes investigaciones sobre estudios exploratorios para evaluar los conocimientos y habilidades de los estudiantes en matemáticas, a partir de esta revisión se diseñó el instrumento a utilizar; 2) se planeó la aplicación del instrumento y se midió la objetividad del mismo; 3) se aplicó el instrumento a 225 estudiantes y, 4) se elaboró la matriz de datos para su posterior análisis.

En la primera etapa se conformó un grupo de cinco profesores que participan en el proceso de rediseño curricular de la carrera de ingeniería en comunicaciones y electrónica (ICE), que cuentan con amplia experiencia en la impartición del curso de Cálculo Diferencial e Integral correspondiente al primer semestre de la carrera. Debido a su experiencia en la impartición de diferentes cursos de matemáticas, estos profesores conocen las deficiencias de los estudiantes y los prerrequisitos de asignaturas subsecuentes, tanto de matemáticas como de materias de especialidad. Los profesores solicitaron la asignatura de Cálculo Diferencial e Integral en el semestre agosto diciembre de 2016, para garantizar la aplicación del instrumento el primer día de clases a por lo menos 8 grupos de los 17 de primer ingreso. Para el diseño del instrumento se revisaron 
trabajos sobre las pruebas para evaluar los conocimientos y habilidades en matemáticas, Kantrov (2000) explica que estas pruebas proporcionan información relacionada con el tipo de audiencias y propósitos para los que son utilizadas. Atendiendo al propósito, clasifica las pruebas en dos grandes grupos: (1) para mejorar el proceso de aprendizaje en las aulas, y (2) para proporcionar información fiable a estudiantes, profesores y directivos de las escuelas, con el propósito de que sean corresponsables de los aprendizajes de los estudiantes y puedan ser utilizados en los procesos de diseño o (re)diseño curricular. En este último grupo se encuentran las pruebas de opción múltiple, utilizadas para conocer el dominio que tienen los estudiantes de un contenido específico en comparación con el desempeño de otros estudiantes, Kantrov (2000) menciona que la importancia de estas pruebas va más allá de la puntuación que en ellas se obtiene, ya que brinda información del contenido matemático implícito en la prueba. Las pruebas de opción múltiple pueden servir también para conocer sobre estructuras específicas de conocimiento y habilidades de los estudiantes, los que brinda información sobre sus fortalezas y debilidades cognitivas (Leighton y Gierl, 2007).

Los profesores analizaron diferentes instrumentos (pruebas de opción múltiple) que evalúan los niveles de dominio de los estudiantes en matemáticas, en el último año de bachillerato o el primer año de universidad (INEE, 2011; Tirado, et al., 1997; González, 2009). De la revisión de los instrumentos, se identificó una gran similitud en las características de la población que participaría en el estudio, con las reportadas por González (2009). Las áreas incluidas en el estudio realizado por el Consejo Regional del Área Metropolitana y la Asociación Nacional de Universidades e Instituciones de Educación Superior (CRAM-ANUIES), también fueron consideradas en este estudio como requisitos en la formación básica de los estudiantes que ingresan a las carreras de ingeniería. Este fue el segundo criterio para utilizar el instrumento diseñado por el CRAMANUIES, como base para el diseño del que fue aplicado en la presente investigación.

En el estudio realizado por el CRAM-ANUIES (González, 2009), se reportan los resultados de un diagnóstico sobre los conocimientos y habilidades en matemáticas de los estudiantes que ingresan a las instituciones de educación superior, y su relación con indicadores de carácter socio familiar y de formación previa. En el estudio participaron estudiantes de primer semestre de educación superior de diez instituciones públicas y privadas de la zona metropolitana de la Ciudad de México. El instrumento consta de dos partes, la primera formulada para obtener información de carácter socio familiar y de su formación previa, la segunda orientada para obtener información sobre el nivel de conocimientos y habilidades en matemáticas en cuatro áreas básicas: manejo de información, álgebra, aritmética y geometría.

\section{El instrumento}

Además de las áreas básicas incluidas por el CRAM-ANUIES (González, 2009), el grupo de profesores consideraron necesario considerar también las áreas de geometría analítica, cálculo diferencial y cálculo integral por ser incluidas en el examen de admisión a la institución. De esta forma el instrumento quedó conformado por dos secciones, la primera contiene la información de cada estudiante, la segunda incluye 40 ítems que corresponden a siete áreas de conocimientos de matemáticas: ocho ítems de álgebra, ocho de geometría, ocho de aritmética, ocho de manejo de información, tres de geometría analítica, tres de cálculo diferencial y dos de cálculo integral. Los ítems se clasificaron (tabla 1) utilizando la taxonomía de Stein et al. (2009). El grupo de profesores determinó que no serían incluidos ítems que se ubicaran en el nivel alto de demanda cognitiva, en el que el trabajo se orienta al uso de teoremas y demostraciones. La decisión se tomó debido a que se contaría con 1 hora 30 minutos para la aplicación del instrumento, y el incluir ítems de este nivel de demanda requería de más tiempo para su solución.

Tabla 1. Clasificación de los ítems utilizando la taxonomía de Stein et al. (2009)

\begin{tabular}{|c|c|c|c|c|c|c|c|}
\hline & \multicolumn{7}{|c|}{ Nivel de demanda cognitiva en el que se privilegia la memorización } \\
\hline & Álgebra & Geometría & Aritmética & $\begin{array}{l}\text { Manejo de } \\
\text { información }\end{array}$ & $\begin{array}{c}\text { Geometría } \\
\text { Analítica }\end{array}$ & $\begin{array}{l}\text { Cálculo } \\
\text { diferencial }\end{array}$ & $\begin{array}{l}\text { Cálculo } \\
\text { integral }\end{array}$ \\
\hline $\begin{array}{l}\text { No. de } \\
\text { ítem }\end{array}$ & $1,4,5$ & $9,10,11,12$ & $17,23,24$ & 25 & 33,35 & 36 & 40 \\
\hline \multirow[t]{3}{*}{$\begin{array}{l}\text { Total de } \\
\text { ítems }\end{array}$} & 3 & 4 & 3 & 1 & 2 & 1 & 1 \\
\hline & \multicolumn{7}{|c|}{ Nivel de demanda cognitiva, con énfasis en procedimientos sin conexiones entre ellos } \\
\hline & Álgebra & Geometría & Aritmética & $\begin{array}{l}\text { Manejo de } \\
\text { información }\end{array}$ & $\begin{array}{c}\text { Geometría } \\
\text { Analítica }\end{array}$ & $\begin{array}{l}\text { Cálculo } \\
\text { diferencial }\end{array}$ & $\begin{array}{l}\text { Cálculo } \\
\text { integral }\end{array}$ \\
\hline
\end{tabular}


Tabla 1 (continuación)

\begin{tabular}{|c|c|c|c|c|c|c|c|}
\hline $\begin{array}{l}\text { No. De } \\
\text { ítem }\end{array}$ & 2,3 & 13,14 & $18,20,22$ & $\begin{array}{c}26,27,28 \\
29,30\end{array}$ & 34 & 37 & 0 \\
\hline \multirow[t]{3}{*}{$\begin{array}{l}\text { Total de } \\
\text { ítems }\end{array}$} & 2 & 2 & 3 & 5 & 1 & 1 & 0 \\
\hline & \multicolumn{7}{|c|}{ Nivel de demanda cognitiva, con énfasis en procedimientos con conexiones entre ellos } \\
\hline & Álgebra & Geometría & Aritmética & $\begin{array}{l}\text { Manejo de } \\
\text { información }\end{array}$ & $\begin{array}{c}\text { Geometría } \\
\text { Analítica }\end{array}$ & $\begin{array}{l}\text { Cálculo } \\
\text { diferencial }\end{array}$ & $\begin{array}{l}\text { Cálculo } \\
\text { integral }\end{array}$ \\
\hline $\begin{array}{l}\text { No. de } \\
\text { ítem }\end{array}$ & $6,7,8$ & 15,16 & 19,21 & 31,32 & 0 & 38 & 39 \\
\hline $\begin{array}{l}\text { Total de } \\
\text { ítems }\end{array}$ & 3 & 2 & 2 & 2 & 0 & 1 & 1 \\
\hline
\end{tabular}

En el nivel de demanda cognitiva (en el que se privilegia la memorización) se colocaron 15 ítems, 1 ítem dedicado a cálculo integral y 1 a cálculo diferencial, mientras que se incluyeron 4 en áreas como geometría. Esto fue debido a que a partir del Ciclo Escolar 2009-2010, en México, la Dirección General de Bachillerato realizó la Reforma Integral de la Educación Media Superior, en la que se identifican los niveles de complejidad deseables para aquellos estudiantes que opten por una determinada trayectoria académica (DOF, 2009). En las asignaturas de cálculo diferencial y de cálculo integral se espera que los estudiantes desarrollen los conocimientos y las habilidades básicas que son necesarias para el área de ciencias físico matemáticas, por lo que al ser asignaturas opcionales, no todos los estudiantes están obligados a cursarlas.

Un ítem ubicado en el nivel de demanda cognitiva en el que se privilegia la memorización es el ítem 5. Instrucciones. Simplifica la siguiente expresión $-x^{2}+9 x-20$. El resultado que obtienes es:
a) $2 x-8$
b) $x-2$
c) $x-8$
d) $8-2 x$

Comentario. Este ítem requiere de la aplicación de una regla previamente aprendida, que se incluye de manera implícita en el enunciado. Los estudiantes tienen que efectuar la operación indicada respetando los signos de agrupación; el ítem incluye un anidamiento por lo que se deben realizar las operaciones indicadas del signo más interno al externo. Aun cuando los estudiantes han trabajado con este tipo de tareas durante el bachillerato, muestran dificultades en ellas, Booth (1988) señala que las dificultades que muestran los estudiantes en Álgebra tienen que ver con la naturaleza de las respuestas, el centro está en los procedimientos y relaciones, sin embargo, muchos estudiantes asumen que la respuesta que se requiere es numérica. En el uso de paréntesis también se encuentran dificultades, algunas debidas a que los estudiantes consideran que la secuencia de lo escrito determina el orden en el que las operaciones deben ser ejecutadas, lo que determina errores que pueden estar relacionados con una visión incorrecta de la forma de ejecutar operaciones aritméticas que también incluyen signos de agrupación (Kieran, 1979). Greens y Rubinstein (2008) señalan que los estudiantes desde los grados 8 y 9 muestran dificultades con algunos conceptos y habilidades algebraicas, y en no pocos casos, esta es una posible causa para que eviten los cursos de matemáticas superiores.

El Ítem 20 se ubica en el nivel de demanda cognitiva con énfasis en los procedimientos sin conexiones. Instrucciones. En un depósito que estaba lleno se han sacado $\frac{2}{3}$ del total, y después $\frac{1}{5}$ del total. Sabiendo que aún quedan 400 litros, ¿cuál era la capacidad del depósito?:
a) 400 litros
b) 900 litros
c) 2000 litros
d) 3000 litros

Comentario. Para resolver con éxito este ítem se utiliza un algoritmo, un procedimiento que es evidente de una experiencia anterior. Los estudiantes tienen que calcular el total del que se ha tomado de una parte utilizando operaciones con fracciones. La solución de este tipo de problemas tiene que ver con las experiencias de aprendizaje con las fracciones, que han tenido los estudiantes en sus cursos anteriores; estas son consideradas como elementos de infinitas clases de equivalencia que forman a los números racionales (Behr et al., 1992). Para Freudenthal (1973) las fracciones son el origen fenomenológico del concepto de número racional y tienen cuatro aspectos fundamentales a considerar. El tercer aspecto se refiere a la noción de parte de un todo o la comparación de diferentes partes de un todo y es la que se pone en juego en el ítem 20. 
El ítem 31 se ubica en el nivel de demanda cognitiva en el que se pone énfasis en los procedimientos y las conexiones entre ellos.

Instrucciones. La figura representa la distribución porcentual de horas a la semana que destina un estudiante a actividades cotidianas.

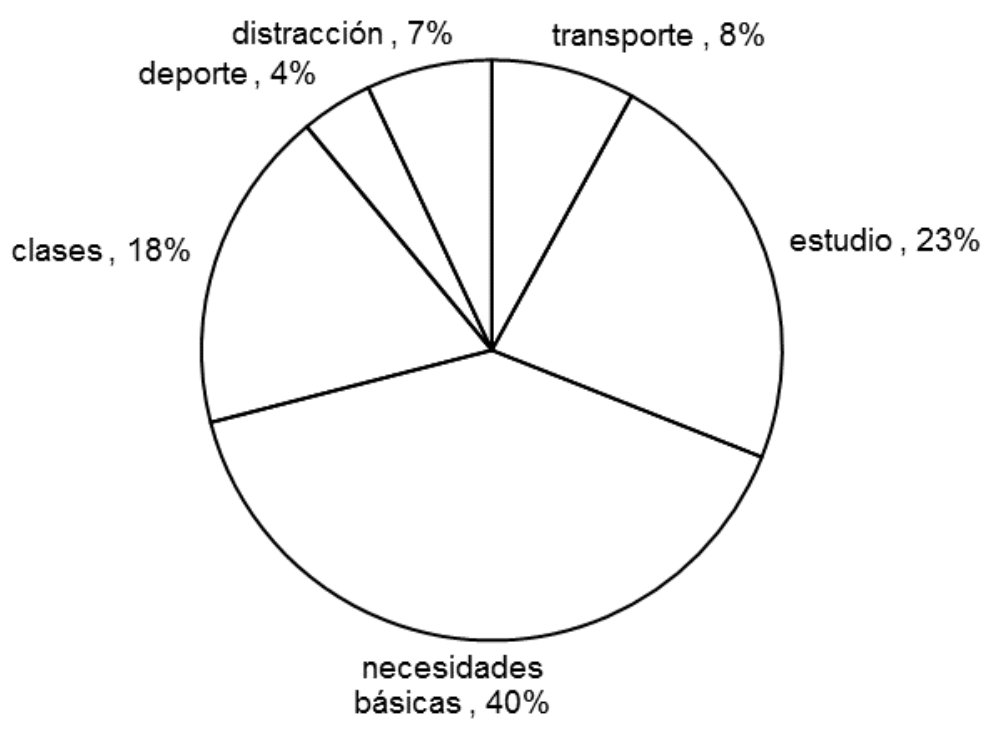

Fig. 1: Esquema correspondiente al ítem 31 del instrumento usado. (Adaptada de González, 2009)

Instrucciones. La medida en grados del ángulo central del sector correspondiente a las clases es:
a) $64.8^{\circ}$
b) $11.5^{\circ}$
c) $32.4^{\circ}$
d) 120

Comentario. Este ítem se ubica en el nivel alto de demanda cognitiva, procedimiento con conexiones; sugiere trayectorias explícitas o implícitas a seguir que son procedimientos generales que tienen conexión con los conceptos e ideas subyacentes. El concepto de ángulo central, permitirá dar respuesta a la pregunta, este tema se encuentra incluido en los programas de la educación secundaria, en el eje temático de Forma Espacio y Medida; en el que se estudian y aplican propiedades de diversos elementos del círculo, como circunferencia, superficie, ángulo inscrito y central, arcos de la circunferencia y sectores circulares (SEP, 2011). El estudio de la geometría se considera como uno de los pilares en la formación académica de las personas, al contribuir al desarrollo de diferentes habilidades propias del quehacer dentro de la matemática que pueden ser utilizadas en diferentes contextos en los que la geometría tiene aplicación (Jones, 2002; Corica y Marín, 2014).

En la segunda etapa los cinco profesores que participaron en el proyecto realizaron la planeación para aplicar el instrumento el primer día de clases del semestre agosto diciembre de 2016. Verificaron el tiempo que les tomaría a los estudiantes dar respuesta al instrumento; realizaron la reproducción del cuestionario y de las hojas de respuesta; realizaron la distribución de los materiales a los profesores que aceptaron participar en la aplicación del instrumento el primer día de clases; acordaron la construcción de la base de datos y el análisis de la misma en la versión 22 del software SPSS y en la hoja de cálculo Excel de Microsoft-Office; utilizaron los indicadores: nivel de dificultad y poder de discriminación para evaluar la objetividad del instrumento.

La validación del instrumento se realizó a través de la prueba para consistencia interna de Kuder-Richardson (KR) (Campos-Arias y Oviedo, 2008). Se aplicó esta prueba dado que el tipo de respuesta del instrumento es dicotómica: correcta o incorrecta, para los 40 ítems. El valor de $K R=0.77$ se considera aceptable para la consistencia interna del instrumento (Campos-Arias y Oviedo, 2008). A su vez las correlaciones del dominio total definido como número de aciertos presentaron correlaciones significativas, $\rho<0.05$, para cada uno de los ítems en las tres dimensiones: memorización, procedimiento sin conexiones y procedimiento con conexiones.

\section{La dificultad de los ítems}

La dificultad se refiere a la proporción de personas que responden correctamente un ítem de una prueba. Entre mayor sea esta proporción, menor será su dificultad, a mayor dificultad del ítem, menor será su índice. La dificultad de cada ítem se calculó dividiendo el número de personas que contestó correctamente el ítem entre el número total de personas que lo respondieron (Backhoff et al., 2000). 


$$
\begin{aligned}
& p_{i}=\frac{A_{i}}{T} \\
& p_{i}=\text { índice de dificutad del reactivo } i \\
& A_{i}=\text { número de aciertos en el reactivo } i \\
& T=\text { número de personas que respondieron el reactivo } i
\end{aligned}
$$

\section{La discriminación}

La discriminación permite establecer una diferencia entre aquellos estudiantes que obtuvieron buenas calificaciones en la prueba y aquellos que obtuvieron bajas calificaciones. Es decir, si un instrumento y un ítem miden la misma habilidad, se espera que quien tuvo una puntuación alta en todo el instrumento tendrá altas probabilidades de contestar correctamente el ítem.

$$
\begin{aligned}
& D=\frac{G A-G B}{N} \\
& G A=25 \% \text { de personas que respondieron correctamente el reactivo con el puntaje más alto } \\
& G B=25 \% \text { de personas que respondieron correctamente el reactivo con el puntaje más bajo } \\
& N=\text { número de personas del grupo más numeroso }
\end{aligned}
$$

Tercera etapa. En la tercera etapa se llevó a cabo la aplicación del instrumento a 225 estudiantes, inscritos en primer semestre de una carrera de ingeniería en el periodo agosto-diciembre de 2016. En el estudio participaron 134 hombres y 91 mujeres. La aplicación del instrumento tuvo una duración de 1 hora 30 minutos y se llevó a cabo el primer y segundo día de clases. Posteriormente, se construyó la base que fue analizada como se describe en la siguiente y última etapa.

Cuarta etapa. Para registrar las respuestas de cada estudiante en cada uno de los ítems, se utilizó una variable dicotómica $y_{j}$ que toma el valor 1 si la respuesta al ítem $i$ es correcta y 0 en caso contrario. De tal forma que un vector $x_{i}$ de la matriz de datos, contiene las respuestas del estudiante $i$ a cada uno de los ítems $y_{j}$. Se capturó la información en el paquete estadístico SPSS versión 19 y los datos se analizaron con apoyo de estadística descriptiva.

\section{RESULTADOS Y ANÁLISIS}

Con los datos obtenidos se determinó el número de estudiantes que resolvieron con éxito los ítems de cada nivel de demanda cognitiva (tabla 1) y se identificaron aquellos ítems que tuvieron mayor dificultad para los estudiantes. En cada nivel los resultados se agruparon en bloques, en color blanco se presentan los ítems con los porcentajes más altos de respuestas correctas (de $80 \%$ a $100 \%$ ); en color gris intenso los ítems con los porcentajes intermedios de respuestas correctas (de 60\% a 79\%); y en gris claro los ítems en los que los estudiantes mostraron mayor dificultad (de $0 \%$ a $59 \%$ ).

\section{Ítems del nivel de demanda cognitiva en el que se privilegia la memorización}

En la tabla 2 se condensan las respuestas correctas e incorrectas que obtuvieron los hombres y mujeres en cada uno de los ítems del instrumento, clasificados con un nivel de demanda cognitiva en el que se privilegia la memorización; las siglas utilizadas son: A: álgebra; G: geometría; ART: aritmética; MI: manejo de información; GA: geometría analítica; CD: cálculo diferencial y Cl: cálculo integral. En este nivel, el valor mínimo de respuestas correctas fue de 70 (ítem 36) y el máximo de 202 (ítem 17), obteniéndose un rango de 132. Se tomó como promedio de respuestas correctas la mediana con un valor de 158.

De los ítems de este nivel de demanda cognitiva, los porcentajes más altos de respuestas correctas, tanto de hombres como de mujeres se identificaron en los ítems 17 de Aritmética, 24 de Aritmética y 9 de Geometría con $89.8 \%$, $85.3 \%$ y $83.1 \%$ respectivamente (marcados en blanco en la tabla 2 ); las respuestas de los estudiantes en estos ítems indican un adecuado manejo de porcentajes, operaciones con potencias y aplicación del Teorema de Pitágoras. Los porcentajes intermedios se identificaron en los ítems 11 de Geometría, 5 de Álgebra, 1 de Álgebra, 25 de Manejo de Información, 4 de Álgebra y 35 de Geometría Analítica, con porcentajes de respuestas correctas de 78.7\%, 76.9\%, 72.9\%, 70.7\%, 70.2\% y 68.4\% respectivamente (marcados en gris intenso en la tabla 2); los temas incluidos en estos ítems corresponden al cálculo de áreas, factorización de trinomios, solución de ecuaciones de primer grado, interpretación de una gráfica, simplificación de una expresión algebraica y la relación entre la gráfica de una función y su ecuación. 
En los ítems 12 de Geometría Analítica, 10 de Geometría, 40 de Cálculo Integral, 23 de Aritmética, 33 de Geometría Analítica y 36 de Cálculo Diferencial se identificaron los porcentajes más altos de respuestas incorrectas, con $44.9 \%, 51.1 \%, 51.6 \%, 58.7 \%, 58.7 \%$ y $68.9 \%$, respectivamente (marcados en gris claro en la tabla 2); la diferencia en los porcentajes obtenidos por hombres y mujeres en estos ítems osciló en 10 puntos porcentuales. Los temas incluidos en estos ítems fueron cálculo de volúmenes, cálculo de integrales indefinidas, operaciones aritméticas de suma y resta de números enteros, manejo de signos de agrupación, ecuación de una recta y derivadas.

Como ya se mencionó, en relación con el desempeño global de los estudiantes, los porcentajes más altos de respuestas correctas corresponden a los ítems 17, 24 y 9, con porcentajes mayores al 83\%. El porcentaje de respuestas correctas en los hombres osciló entre $89.6 \%$ y $91.8 \%$, mientras que en las mujeres entre $73.6 \%$ y $89 \%$. Se identificó que el cálculo de potencias de números enteros (ítem 24) tuvo mayor dificultad para las mujeres que para los hombres; a diferencia del ítem 17 en el que el porcentaje de respuestas correctas casi es el mismo. Un comportamiento más homogéneo entre hombres y mujeres se identificó en los porcentajes intermedios de respuestas correctas; en los hombres osciló entre $68.7 \%$ y $81.3 \%$, en las mujeres entre $65.9 \%$ y $75.8 \%$. Con los datos de la Tabla 2 es posible identificar similitud en el desempeño de los hombres y mujeres en el ítem 35 de Geometría Analítica correspondiente a la ecuación de la parábola. También se identificó que para los hombres la simplificación de una expresión algebraica tuvo menor dificultad que para las mujeres, como se observa en las respuestas al ítem 4 de Álgebra.

Tabla 2. Tabla de contingencia por género

\begin{tabular}{|c|c|c|c|c|c|c|c|}
\hline \multicolumn{8}{|c|}{ Nivel de demanda cognitiva en el que se privilegia la memorización } \\
\hline \multirow{2}{*}{ Ítem } & \multicolumn{2}{|c|}{ Correcto } & \multicolumn{2}{|c|}{ Incorrecto } & \multirow{2}{*}{$\begin{array}{c}\text { Total } \\
\text { Correcto }\end{array}$} & \multirow{2}{*}{$\begin{array}{c}\text { Total } \\
\text { Incorrecto }\end{array}$} & \multirow{2}{*}{ Total } \\
\hline & $\mathrm{H}$ & M & $\mathrm{H}$ & M & & & \\
\hline $1 \mathrm{~A}$ & $\begin{array}{c}103 \\
76.9 \%\end{array}$ & $\begin{array}{c}61 \\
67.0 \%\end{array}$ & $\begin{array}{c}31 \\
23.1 \%\end{array}$ & $\begin{array}{c}30 \\
33.0 \%\end{array}$ & $\begin{array}{c}164 \\
72.9 \%\end{array}$ & $\begin{array}{c}91 \\
27.1 \%\end{array}$ & $\begin{array}{c}225 \\
100 \%\end{array}$ \\
\hline $4 \mathrm{~A}$ & $\begin{array}{c}98 \\
73.1 \%\end{array}$ & $\begin{array}{c}60 \\
65.9 \%\end{array}$ & $\begin{array}{c}36 \\
26.9 \%\end{array}$ & $\begin{array}{c}31 \\
34.1 \%\end{array}$ & $\begin{array}{c}158 \\
70.2 \%\end{array}$ & $\begin{array}{c}67 \\
29.8 \%\end{array}$ & $\begin{array}{c}225 \\
100 \%\end{array}$ \\
\hline $5 \mathrm{~A}$ & $\begin{array}{c}109 \\
81.3 \%\end{array}$ & $\begin{array}{c}64 \\
70.3 \%\end{array}$ & $\begin{array}{c}25 \\
18.7 \%\end{array}$ & $\begin{array}{c}27 \\
29.7 \%\end{array}$ & $\begin{array}{c}173 \\
76.9 \%\end{array}$ & $\begin{array}{c}52 \\
23.1 \%\end{array}$ & $\begin{array}{c}225 \\
100 \%\end{array}$ \\
\hline $9 G$ & $\begin{array}{c}120 \\
89.6 \%\end{array}$ & $\begin{array}{c}67 \\
73.6 \%\end{array}$ & $\begin{array}{c}14 \\
10.4 \%\end{array}$ & $\begin{array}{c}24 \\
26.4 \%\end{array}$ & $\begin{array}{c}187 \\
83.1 \%\end{array}$ & $\begin{array}{c}38 \\
16.9 \%\end{array}$ & $\begin{array}{c}225 \\
100 \%\end{array}$ \\
\hline $10 \mathrm{G}$ & $\begin{array}{c}72 \\
53.7 \%\end{array}$ & $\begin{array}{c}38 \\
41.8 \%\end{array}$ & $\begin{array}{c}62 \\
46.3 \%\end{array}$ & $\begin{array}{c}53 \\
58.2 \%\end{array}$ & $\begin{array}{c}110 \\
48.9 \%\end{array}$ & $\begin{array}{c}115 \\
51.1 \%\end{array}$ & $\begin{array}{c}225 \\
100 \%\end{array}$ \\
\hline $11 G$ & $\begin{array}{c}108 \\
80.6 \%\end{array}$ & $\begin{array}{c}699 \\
75.8 \%\end{array}$ & $\begin{array}{c}26 \\
19.4 \%\end{array}$ & $\begin{array}{c}22 \\
24.2 \%\end{array}$ & $\begin{array}{c}177 \\
78.7 \%\end{array}$ & $\begin{array}{c}48 \\
21.3 \%\end{array}$ & $\begin{array}{c}225 \\
100 \%\end{array}$ \\
\hline $12 \mathrm{G}$ & $\begin{array}{c}75 \\
56.0 \%\end{array}$ & $\begin{array}{c}49 \\
53.8 \%\end{array}$ & $\begin{array}{c}59 \\
44.0 \%\end{array}$ & $\begin{array}{c}42 \\
46.2 \%\end{array}$ & $\begin{array}{c}124 \\
55.1 \%\end{array}$ & $\begin{array}{c}101 \\
44.9 \%\end{array}$ & $\begin{array}{c}225 \\
100 \%\end{array}$ \\
\hline 17ART & $\begin{array}{c}121 \\
90.3 \%\end{array}$ & $\begin{array}{c}81 \\
89.0 \%\end{array}$ & $\begin{array}{c}13 \\
9.7 \%\end{array}$ & $\begin{array}{c}10 \\
11.0 \%\end{array}$ & $\begin{array}{c}202 \\
89.8 \%\end{array}$ & $\begin{array}{c}23 \\
10.2 \%\end{array}$ & $\begin{array}{c}225 \\
100 \%\end{array}$ \\
\hline 23ART & $\begin{array}{c}61 \\
45.5 \%\end{array}$ & $\begin{array}{c}32 \\
35.2 \%\end{array}$ & $\begin{array}{c}73 \\
54.5 \%\end{array}$ & $\begin{array}{c}59 \\
64.8 \%\end{array}$ & $\begin{array}{c}93 \\
41.3 \%\end{array}$ & $\begin{array}{c}132 \\
58.7 \%\end{array}$ & $\begin{array}{c}225 \\
100 \%\end{array}$ \\
\hline 24ART & $\begin{array}{c}123 \\
91.8 \%\end{array}$ & $\begin{array}{c}69 \\
75.8 \%\end{array}$ & $\begin{array}{c}11 \\
8.2 \%\end{array}$ & $\begin{array}{c}22 \\
24.2 \%\end{array}$ & $\begin{array}{c}192 \\
85.3 \%\end{array}$ & $\begin{array}{c}33 \\
14.7 \%\end{array}$ & $\begin{array}{c}225 \\
100 \%\end{array}$ \\
\hline $25 \mathrm{Ml}$ & $\begin{array}{c}97 \\
72.4 \%\end{array}$ & $\begin{array}{c}62 \\
68.1 \%\end{array}$ & $\begin{array}{c}37 \\
27.6 \%\end{array}$ & $\begin{array}{c}29 \\
68.1 \% \\
\end{array}$ & $\begin{array}{c}159 \\
70.7 \% \\
\end{array}$ & $\begin{array}{c}66 \\
29.3 \% \\
\end{array}$ & $\begin{array}{c}225 \\
100 \% \\
\end{array}$ \\
\hline 33GA & $\begin{array}{c}63 \\
47.0 \%\end{array}$ & $\begin{array}{c}30 \\
33.0 \%\end{array}$ & $\begin{array}{c}71 \\
53.0 \%\end{array}$ & $\begin{array}{c}61 \\
67.0 \%\end{array}$ & $\begin{array}{c}93 \\
41.3 \%\end{array}$ & $\begin{array}{c}132 \\
58.7 \%\end{array}$ & $\begin{array}{c}225 \\
100 \%\end{array}$ \\
\hline 35GA & $\begin{array}{c}92 \\
68.7 \%\end{array}$ & $\begin{array}{c}62 \\
68.1 \%\end{array}$ & $\begin{array}{c}42 \\
31.3 \%\end{array}$ & $\begin{array}{c}29 \\
31.9 \%\end{array}$ & $\begin{array}{c}154 \\
68.4 \%\end{array}$ & $\begin{array}{c}71 \\
31.6 \%\end{array}$ & $\begin{array}{c}225 \\
100 \%\end{array}$ \\
\hline $36 \mathrm{CD}$ & $\begin{array}{c}47 \\
35.1 \%\end{array}$ & $\begin{array}{c}23 \\
25.3 \%\end{array}$ & $\begin{array}{c}87 \\
64.9 \%\end{array}$ & $\begin{array}{c}68 \\
74.7 \%\end{array}$ & $\begin{array}{c}70 \\
31.1 \%\end{array}$ & $\begin{array}{c}155 \\
68.9 \%\end{array}$ & $\begin{array}{c}225 \\
100 \%\end{array}$ \\
\hline $40 \mathrm{Cl}$ & $\begin{array}{c}70 \\
52.2 \%\end{array}$ & $\begin{array}{c}39 \\
42.9 \%\end{array}$ & $\begin{array}{c}64 \\
47.8 \%\end{array}$ & $\begin{array}{c}52 \\
57.1 \%\end{array}$ & $\begin{array}{c}109 \\
48.4 \%\end{array}$ & $\begin{array}{c}116 \\
51.6 \%\end{array}$ & $\begin{array}{c}194 \\
86.2 \%\end{array}$ \\
\hline
\end{tabular}




\section{Ítems en el nivel de demanda cognitiva en el que se privilegian los procedimientos sin conexiones}

En la tabla 3 se presentan los resultados correspondientes al nivel de demanda cognitiva en el que se privilegian los procedimientos sin conexiones. En este nivel de demanda cognitiva, el valor mínimo de respuestas correctas fue de 49 (ítem 37) y el máximo de 209 (ítem 2), con un rango de 160. Como promedio de respuestas correctas se tomó la mediana con un valor de 168. En este bloque los porcentajes más altos de respuestas correctas, tanto de hombres como de mujeres, se identificaron en los ítems 2 de Álgebra, 26 de Manejo de Información, 18 de Aritmética y 28 de Manejo de Información, con 92.9\%, 92\% y 89.3\% y 81.8\% respectivamente (marcados en blanco en la tabla 3). Las respuestas de los estudiantes en estos ítems indican que la mayoría de ellos pueden representar un enunciado verbal en forma algebraica y calcular porcentajes para dar solución a problemas verbales. Los porcentajes intermedios se identificaron en los ítems 22 de Aritmética, 13 de Geometría, 14 de Geometría y 27 de Manejo de Información, con porcentajes de respuestas correctas de $79.1 \%, 78.2 \%, 75.6 \%, 74.2 \%$, respectivamente (marcados en gris intenso en la tabla 3 ). Los temas incluidos en estos ítems corresponden a cálculo de razones y proporciones, cálculo de áreas y propiedades de triángulos y cálculo de porcentajes para dar solución a problemas verbales.

Las mayores dificultades se presentaron en los ítems: 37 de Cálculo Diferencial, 3 de Álgebra, 34 de Geometría Analítica, 20 de Aritmética, 30 de Manejo de Información y 29 de Manejo de Información, con los porcentajes de respuestas incorrectas de $78.2 \%, 62.2 \%, 60.9 \%, 50.7 \%, 45.8 \%$ y $45.3 \%$ respectivamente (marcados en gris claro en la tabla 3 ). En estos ítems la diferencia en los porcentajes obtenidos por hombres y mujeres fue menor a 8 puntos porcentuales. Los temas incluidos en estos ítems fueron, interpretación geométrica de la derivada de una función real de variable real, solución de una ecuación con una condición dada, definición de circunferencia como lugar geométrico, resolución de problemas verbales mediante una ecuación de primer grado y nociones de probabilidad.

El porcentaje de respuestas correctas en los hombres osciló entre $85.1 \%$ y $95.5 \%$, mientras que en las mujeres entre $76.9 \%$ y $90.1 \%$. Se identifica que en este bloque el ítem 2 de Álgebra fue el de menor dificultad para los hombres, no así para las mujeres, quienes presentaron menores dificultades en el ítem 26 de Manejo de Información. En los porcentajes intermedios de respuestas correctas se identificó que mientras que para los hombres los ítems 13 y 14 de Geometría fueron los de menor dificultad, para las mujeres fueron el 22 de Aritmética y el 27 de Manejo de Información. En los ítems que presentaron mayor dificultad se identificó un comportamiento similar tanto en hombres como en mujeres, como se observa en el ítem 37 de Cálculo Diferencial; y una mayor dificultad en las mujeres en comparación con el desempeño de los hombres, en el ítem 3 de Álgebra.

Tabla 3. Tabla de contingencia por género

\begin{tabular}{|c|c|c|c|c|c|c|c|}
\hline \multirow{2}{*}{ Ítem } & \multicolumn{2}{|c|}{ Correcto } & \multicolumn{2}{|c|}{ Incorrecto } & \multirow{2}{*}{$\begin{array}{c}\text { Total } \\
\text { Correcto }\end{array}$} & \multirow{2}{*}{$\begin{array}{c}\text { Total } \\
\text { Incorrecto }\end{array}$} & \multirow{2}{*}{ Total } \\
\hline & $\mathrm{H}$ & $M$ & $\mathrm{H}$ & $M$ & & & \\
\hline $2 A$ & $\begin{array}{c}128 \\
95.5 \%\end{array}$ & $\begin{array}{c}81 \\
89.0 \%\end{array}$ & $\begin{array}{c}6 \\
4.5 \%\end{array}$ & $\begin{array}{c}10 \\
11.0 \%\end{array}$ & $\begin{array}{c}209 \\
92.9 \%\end{array}$ & $\begin{array}{c}16 \\
7.1 \%\end{array}$ & $\begin{array}{c}225 \\
100 \%\end{array}$ \\
\hline $3 A$ & $\begin{array}{c}58 \\
43.3 \%\end{array}$ & $\begin{array}{c}27 \\
29.7 \%\end{array}$ & $\begin{array}{c}76 \\
56.7 \%\end{array}$ & $\begin{array}{c}64 \\
70.3 \%\end{array}$ & $\begin{array}{c}85 \\
37.8 \%\end{array}$ & $\begin{array}{c}140 \\
62.2 \%\end{array}$ & $\begin{array}{c}225 \\
100 \%\end{array}$ \\
\hline $13 G$ & $\begin{array}{c}113 \\
84.3 \%\end{array}$ & $\begin{array}{c}63 \\
69.2 \%\end{array}$ & $\begin{array}{c}21 \\
15.7 \%\end{array}$ & $\begin{array}{c}28 \\
30.8 \%\end{array}$ & $\begin{array}{c}176 \\
78.2 \%\end{array}$ & $\begin{array}{c}49 \\
21.8 \%\end{array}$ & $\begin{array}{c}225 \\
100 \%\end{array}$ \\
\hline $14 G$ & $\begin{array}{c}108 \\
80.6 \%\end{array}$ & $\begin{array}{c}62 \\
68.1 \%\end{array}$ & $\begin{array}{c}26 \\
19.4 \%\end{array}$ & $\begin{array}{c}29 \\
31.9 \%\end{array}$ & $\begin{array}{c}170 \\
75.6 \%\end{array}$ & $\begin{array}{c}55 \\
24.4 \%\end{array}$ & $\begin{array}{c}225 \\
100 \%\end{array}$ \\
\hline 18ART & $\begin{array}{c}123 \\
91.8 \%\end{array}$ & $\begin{array}{c}78 \\
85.7 \%\end{array}$ & $\begin{array}{c}11 \\
8.2 \%\end{array}$ & $\begin{array}{c}13 \\
14.3 \%\end{array}$ & $\begin{array}{c}201 \\
89.3 \%\end{array}$ & $\begin{array}{c}24 \\
10.7 \%\end{array}$ & $\begin{array}{c}225 \\
100 \%\end{array}$ \\
\hline 20ART & $\begin{array}{c}77 \\
57.5 \% \\
\end{array}$ & $\begin{array}{c}34 \\
37.4 \% \\
\end{array}$ & $\begin{array}{c}57 \\
42.5 \% \\
\end{array}$ & $\begin{array}{c}57 \\
62.6 \% \\
\end{array}$ & $\begin{array}{c}111 \\
49.3 \% \\
\end{array}$ & $\begin{array}{c}114 \\
50.7 \% \\
\end{array}$ & $\begin{array}{c}225 \\
100 \% \\
\end{array}$ \\
\hline 22ART & $\begin{array}{c}112 \\
83.6 \% \\
\end{array}$ & $\begin{array}{c}66 \\
72.5 \% \\
\end{array}$ & $\begin{array}{c}22 \\
16.4 \% \\
\end{array}$ & $\begin{array}{c}25 \\
27.5 \% \\
\end{array}$ & $\begin{array}{c}178 \\
79.1 \% \\
\end{array}$ & $\begin{array}{c}47 \\
20.9 \% \\
\end{array}$ & $\begin{array}{c}225 \\
100 \% \\
\end{array}$ \\
\hline $26 \mathrm{Ml}$ & $\begin{array}{c}125 \\
93.3 \%\end{array}$ & $\begin{array}{c}82 \\
90.1 \%\end{array}$ & $\begin{array}{c}9 \\
6.7 \%\end{array}$ & $\begin{array}{c}9 \\
9.9 \%\end{array}$ & $\begin{array}{l}207 \\
92 \%\end{array}$ & $\begin{array}{l}207 \\
92 \%\end{array}$ & $\begin{array}{c}225 \\
100 \%\end{array}$ \\
\hline 27MI & $\begin{array}{c}103 \\
76.9 \%\end{array}$ & $\begin{array}{c}64 \\
70.3 \%\end{array}$ & $\begin{array}{c}31 \\
23.1 \%\end{array}$ & $\begin{array}{c}27 \\
29.7 \%\end{array}$ & $\begin{array}{c}167 \\
74.2 \%\end{array}$ & $\begin{array}{c}58 \\
25.8 \%\end{array}$ & $\begin{array}{c}225 \\
100 \%\end{array}$ \\
\hline
\end{tabular}


Tabla 3 (continuación)

\begin{tabular}{|l|c|c|c|c|c|c|c|}
\hline \multirow{2}{*}{$28 \mathrm{MI}$} & 114 & 70 & 20 & 21 & 184 & 41 & 225 \\
& $85.1 \%$ & $76.9 \%$ & $14.9 \%$ & $23.1 \%$ & $81.8 \%$ & $18.2 \%$ & $100 \%$ \\
\hline \multirow{2}{*}{$29 \mathrm{MI}$} & 83 & 40 & 51 & 51 & 123 & 102 & 225 \\
& $61.9 \%$ & $44.4 \%$ & $38.1 \%$ & $55.6 \%$ & $54.7 \%$ & $45.3 \%$ & $100 \%$ \\
\hline \multirow{2}{*}{$30 \mathrm{MI}$} & 83 & 39 & 51 & 52 & 122 & 103 & 225 \\
& $61.9 \%$ & $42.9 \%$ & $38.1 \%$ & $57.1 \%$ & $54.2 \%$ & $45.8 \%$ & $100 \%$ \\
\hline \multirow{2}{*}{$34 \mathrm{GA}$} & 55 & 33 & 79 & 58 & 88 & 137 & 225 \\
& $41.0 \%$ & $36.3 \%$ & $59.0 \%$ & $63.7 \%$ & $39.1 \%$ & $60.9 \%$ & $100 \%$ \\
\hline \multirow{2}{*}{$37 \mathrm{CD}$} & 30 & 19 & 104 & 72 & 49 & 176 & 225 \\
& $22.4 \%$ & $20.9 \%$ & $77.6 \%$ & $79.1 \%$ & $21.8 \%$ & $78.2 \%$ & $100 \%$ \\
\hline
\end{tabular}

Ítems en el nivel de demanda cognitiva en el que se privilegian los procedimientos con conexiones entre ellos

En la Tabla 4 se presentan los resultados correspondientes al nivel de demanda cognitiva en el que se privilegian los procedimientos con conexiones. El valor mínimo, para las respuestas correctas, fue de 65 (ítem 8) y el valor máximo de 174 (ítem 31). Así, el rango fue de 109 y la mediana correspondiente a las respuestas correctas tuvo un valor de 115.

Las mayores dificultades se presentaron en los ítems 8 de Álgebra, con el $71.1 \%$ de respuestas incorrectas y el 38 de Cálculo Diferencial con un $71.9 \%$ de respuestas incorrectas. En el ítem 8 los estudiantes tenían que factorizar un trinomio cuadrado perfecto para identificar los posibles valores de las variables involucradas, lo que pudieron realizar el $65 \%$ de los estudiantes, en contraste en el ítem 5 , ubicado en el nivel de demanda cognitiva en el que se privilegia la memorización, los estudiantes también tenían que factorizar un trinomio con un término común y el $73.8 \%$ de los estudiantes lo hizo en forma correcta. La diferencia en los porcentajes de aciertos, parece estar relacionada con la instrucción explícita del ítem 5, en contraste con el requisito de formar el trinomio cuadrado perfecto para proceder a la factorización del ítem 8.

En el ítem 38 los estudiantes tenían que relacionar la interpretación de la derivada como la pendiente de la recta tangente a la gráfica de una función, con el signo de la derivada de una función en un intervalo $(a, b)$, lo que requiere un conocimiento más conceptual. El ítem 38 tuvo un porcentaje de respuestas correctas similar al del 37, en el que se solicitó el valor de la pendiente de la recta tangente a la gráfica de una función en un valor $\mathrm{x}_{0}$, este último ítem se ubica en el bloque de demanda cognitiva en el que se privilegian los procedimientos sin conexiones. El porcentaje de estudiante que respondieron correctamente el ítem 37 fue de $21.8 \%$ y el ítem 38 de $28.9 \%$. De lo anterior, es posible inferir que las dificultades están relacionadas con una falta de conocimiento por parte de los estudiantes de la interpretación geométrica de la derivada.

También es importante destacar que en este bloque III, los porcentajes de respuestas correctas no fueron mayores al $77.3 \%$, a diferencia de lo que se observa en el bloque II con un porcentaje máximo de $92.9 \%$, y en el bloque I con un porcentaje máximo de $89.8 \%$, lo que se identifica en la tabla 4 por la ausencia de celdas en color blanco.

Tabla 4. Tabla de contingencia por género

Nivel de demanda cognitiva en el que se privilegian los procedimientos con conexión entre ellos

\begin{tabular}{|l|c|c|c|c|c|c|c|}
\hline \multirow{2}{*}{ Ítem } & \multicolumn{2}{|c|}{ Correcto } & \multicolumn{2}{c|}{ Incorrecto } & \multirow{2}{*}{$\begin{array}{c}\text { Total } \\
\text { correcto }\end{array}$} & $\begin{array}{c}\text { Total } \\
\text { Incorrecto }\end{array}$ & Total \\
\cline { 2 - 5 } & $\mathrm{H}$ & $\mathrm{M}$ & $\mathrm{H}$ & $\mathrm{M}$ & & \\
\hline \multirow{2}{*}{$6 \mathrm{~A}$} & 66 & 49 & 68 & 42 & 115 & 110 & 225 \\
& $49.3 \%$ & $53.8 \%$ & $50.7 \%$ & $46.2 \%$ & $51.1 \%$ & $48.9 \%$ & $100 \%$ \\
\hline \multirow{2}{*}{$7 \mathrm{~A}$} & 86 & 51 & 48 & 40 & 137 & 88 & 225 \\
& $64.2 \%$ & $56 \%$ & $35.8 \%$ & $44 \%$ & $60.9 \%$ & $39.1 \%$ & $100 \%$ \\
\hline \multirow{2}{*}{$8 \mathrm{~A}$} & 46 & 19 & 88 & 72 & 65 & 160 & 225 \\
& $34.3 \%$ & $20.9 \%$ & $65.7 \%$ & $79.1 \%$ & $28.9 \%$ & $71.1 \%$ & $100 \%$ \\
\hline \multirow{2}{*}{$15 \mathrm{G}$} & 52 & 27 & 82 & 64 & 79 & 146 & 225 \\
& $38.8 \%$ & $29.7 \%$ & $61.2 \%$ & $70.3 \%$ & $35.1 \%$ & $64.9 \%$ & $100 \%$ \\
\hline \multirow{2}{*}{$16 \mathrm{G}$} & 53 & 30 & 81 & 61 & 83 & 142 & 225 \\
& $39.6 \%$ & $33 \%$ & $60.4 \%$ & $67 \%$ & $36.9 \%$ & $63.1 \%$ & $100 \%$ \\
\hline
\end{tabular}


Tabla 4 (continuación)

\begin{tabular}{|l|c|c|c|c|c|c|c|}
\hline \multirow{2}{*}{ 19ART } & 74 & 33 & 60 & 58 & 107 & 118 & 225 \\
& $55.2 \%$ & $36.3 \%$ & $44.8 \%$ & $63.7 \%$ & $47.6 \%$ & $52.4 \%$ & $100 \%$ \\
\hline \multirow{2}{*}{$21 \mathrm{ART}$} & 102 & 66 & 32 & 25 & 168 & 57 & 225 \\
& $76.1 \%$ & $72.5 \%$ & $23.9 \%$ & $27.5 \%$ & $74.7 \%$ & $25.3 \%$ & $100 \%$ \\
\hline \multirow{2}{*}{$31 \mathrm{MI}$} & 103 & 71 & 31 & 20 & 174 & 51 & 225 \\
& $76.9 \%$ & $78 \%$ & $23.1 \%$ & $22 \%$ & $77.3 \%$ & $22.7 \%$ & $100 \%$ \\
\hline \multirow{2}{*}{$32 \mathrm{MI}$} & 93 & 57 & 41 & 34 & 150 & 75 & 225 \\
& $69.4 \%$ & $62.6 \%$ & $30.6 \%$ & $37.4 \%$ & $66.7 \%$ & $33.3 \%$ & $100 \%$ \\
\hline \multirow{2}{*}{$38 \mathrm{CD}$} & 49 & 16 & 85 & 75 & 65 & 160 & 225 \\
& $36.6 \%$ & $17.6 \%$ & $63.4 \%$ & $82.4 \%$ & $28.9 \%$ & $71.1 \%$ & $100 \%$ \\
\hline \multirow{2}{*}{$39 \mathrm{Cl}$} & 83 & 51 & 51 & 40 & 134 & 91 & 225 \\
& $61.9 \%$ & $56 \%$ & $38.1 \%$ & $44 \%$ & $59.6 \%$ & $40.4 \%$ & $100 \%$ \\
\hline
\end{tabular}

\section{Reflexiones Finales}

Los resultados globales del estudio se organizan en torno a dos aspectos: a) las características de los ítems que presentaron mayor dificultad tanto para los hombres como para las mujeres $y, b$ ) el desempeño de los estudiantes en los ítems de acuerdo con el nivel de demanda cognitiva.

a) Se identificó que los estudiantes que participaron en el estudio, presentaron dificultades en el manejo de los signos de agrupación; en el análisis de expresiones algebraicas: para determinar los valores de un factor en una fracción algebraica y para la identificación de la regla de factorización adecuada; en el manejo de la definición de circunferencia como lugar geométrico; en el cálculo de la derivada de una función racional y en el manejo de la interpretación geométrica de la derivada: como la pendiente de la recta tangente a la gráfica de una función en un punto. En este sentido, la dificultad en el cálculo de la derivada de una función racional podría estar relacionada con las dificultades que mostraron los estudiantes en el manejo y análisis de las expresiones algebraicas. También, se destaca que la interpretación geométrica de la derivada, no forma parte de los conocimientos previos de un gran número de este grupo de estudiantes, a pesar de estar presente en los libros de texto que son utilizados en el bachillerato en México.

b)

En el informe MECD (2016) se reporta que un el $23,4 \%$ de los estudiantes se encuentra en los niveles $<1$ y 1 de desempeño en matemáticas y que responden con éxito preguntas relacionadas con contextos matemáticos que les son familiares. Los resultados de la presente investigación permiten afirmar que las tareas en las que se aplica el teorema de Pitágoras o se realiza el cálculo de porcentajes o cálculo numérico de potencias son contextos familiares para más del $80 \%$ de los estudiantes. En cuanto a los procedimientos, los problemas verbales de aritmética y de manejo de información a partir de una gráfica, resultaron familiares para más del $80 \%$ de los estudiantes. En las tareas que requieren de procedimientos con relación entre ellos, no se identificó que fueran familiares para los estudiantes.

c) En relación con el desempeño de los estudiantes en los ítems orientados al concepto o al procedimiento, un ejemplo se tuvo en las preguntas 39 y 40, ambas de cálculo integral; en la primera se solicitó a los estudiantes que identificaran la expresión que representa el área que se forma encima del eje x y debajo de la gráfica de una función continua en un intervalo cerrado, en la segunda se les pidió que calcularan la integral de una función mediante integración por sustitución o cambio de variable. En el ítem 39, se obtuvieron 134 respuestas correctas y en el 40 , el número de respuestas correctas fue de 109. Es decir, aun cuando para los estudiantes estos no fueron de los ítems con mayor grado de dificultad, pues el $59.6 \%$ y $48.4 \%$ respectivamente eligieron la respuesta correcta brindan información valiosa. El ítem 40 requiere de la aplicación de un procedimiento previamente aprendido, y el 39 de la aplicación de un concepto, en estos ítems se observó que los estudiantes tuvieron más dificultad para aplicar el procedimiento que para relacionar el concepto con la información que se les proporcionó.

\section{CONCLUSIONES}

De acuerdo con los resultados de este estudio, y de su discusión y análisis se pueden extraer las siguientes conclusiones principales:

A partir del análisis del trabajo de los estudiantes en el ítem 40 es posible inferir que los estudiantes pueden aprender conceptos aun cuando no puedan aplicar con éxito reglas y procedimientos.

Es necesario que los profesores y directivos de las instituciones de educación superior incluyan políticas para 
apoyar a los estudiantes que carecen de las habilidades básicas en matemáticas que se requieren para que puedan permanecer con éxito en un programa de ingeniería, de no hacerlo un alto porcentaje de estudiantes estarán en desventaja en las diferentes asignaturas que en su mayoría requieren fluidez y habilidades matemáticas básicas, hecho que coincide con lo reportado por Armstrong, y Croft, (1999).

En este estudio se identificó que las mujeres muestran más habilidades con los ítems correspondientes a manejo de información, y menos en los de álgebra. También se aportan evidencias para afirmar que un foco de atención en ambos grupos (hombres y mujeres) se encuentra en los procedimientos y conceptos básicos relacionados con la derivada de funciones y cónicas.

\section{AGRADECIMIENTOS}

Las autoras agradecen el apoyo del Instituto Politécnico Nacional de México, a través del proyecto con número de registro SIP 20161069.

\section{REFERENCIAS}

Armstrong, P. y A. Croft, Identifying the Learning Needs in Mathematics of Entrants to Undergraduate Engineering Programmes in an English University, doi: 10.1080/03043799908923538, European Journal of Engineering Education, 24 (1), 59-71 (1999)

Artavia, A., El método de jerarquía de atributos: una opción para vincular teorías cognitivas y del aprendizaje con prácticas psicométricas y de enseñanza, ISSN: 1409-4703, Revista Electrónica Actualidades Investigativas en Educación, 11 (1), 1-29 (2011)

Backhoff, E., R. Larrazolo y M. Rosas, Nivel de dificultad y poder de discriminación del Examen de Habilidades y Conocimientos Básicos (EXHCOBA), ISSN: 1607-4041, Revista Electrónica de Investigación Educativa 2(1) 11-29 (2000)

Behr, M., G. Harel, T. Post y R. Lesh, Rational number, ratio and proportion, Grouws (Ed.), Handbook of Research on Mathematics Teaching and Learning, 296-333, Macmillan Publishing, New York, USA (1992)

Booth, L., Children's difficulties in beginning Algebra, en The Ideas of Algebra, K-12, Yearbook, Coxford y Shulte (Eds.), Reston, VA: NCTM, New York, USA (1988)

Campos-Arias, A. y H. Oviedo, Propiedades Psicométricas de una Escala: la consistencia interna, doi: 10.15446/rsap, Revista de Salud Pública, 10(5), 831-839 (2008)

Corica, A. y E. Marín, Actividad de Estudio e Investigación para la enseñanza de nociones de geometría, ISSN: 18871984, Números, 85, 91-114 (2014)

DOF, Acuerdo secretarial Núm. 486 por el que se establecen las competencias disciplinares extendidas del Bachillerato General, Diario Oficial de la Federación. Secretaría de Gobernación, Ciudad de México, México (2009)

Etxeberria, P., E. Alberdi, I. Eguia y M. García, Análisis del Rendimiento Académico en Relación al Perfil de Ingreso del Alumnado e Identificación de Carencias Formativas en Materias Básicas de dos Grados de Ingeniería, ISSN: 0718-5006, Formación Universitaria, 10(4), 67-74 (2017)

Freudenthal, H., Mathematics as an educational task, Reidel, Dordrecht, Netherlands (1973)

Garbin, S., ¿Cómo piensan los estudiantes entre 16 y 20 años el infinito? La influencia de los modelos, las representaciones y los lenguajes matemáticos, ISSN: 2007-6819, Revista Latinoamericana de Investigación en Matemática, 8(2), 169-193 (2005)

Gaylord, J., Professional standards for teaching mathematics, National Council of Teachers of Mathematics (NCTM), VA: Author, Reston, USA (1991)

González, R. (Ed.), Conocimientos y habilidades en matemáticas de los estudiantes de primer ingreso a las Instituciones de Educación Superior del área metropolitana de la Ciudad de México, ANUIES-UAM, Ciudad de México, México (2009)

Greens, C. y R. Rubenstein, 70th Yearbook: Algebra and Algebraic Thinking in School Mathematics, NCTM, New York, USA (2008)

INNE, La educación media superior en México, 1ª Ed., Ciudad de México, México (2011)

Jones, K., Issues in the Teaching and Learning of Geometry, Aspects of Teaching Secondary Mathematics. Perspectives on practice, Haggarty (Ed.), pp. 121-139. Routledge Falmer, London, England (2002)

Kantrov, I., Assessing Students' Mathematics Learning, Education Development Center, New York, USA (2000)

Kieran, C., Children's operational thinking within the context of bracketing and the order of operations. In Proceedings of the Third International Conference for the Psychology of Mathematics Education, 128-133, Coventry, England, 9-14 July (1979)

Leighton, J. y M. Gierl, Defining and Evaluating Models of Cognition Used in Educational Measurement to Make Inferences About Examinees' Thinking Processes, ISSN: 1745-3992 Educational Measurement. Issues and Practice, 26(2), 3-16 (2007) 
MECD, Los resultados de los alumnos en PISA: ciencias, lectura y matemáticas, en PISA 2015. Programa para la Evaluación Internacional de los Alumnos, Subdirección General de Documentación y Publicaciones, 61-94, Madrid, España (2016)

SEP, Publicación de resultados primera aplicación 2015, SEP-INEE, México (2015)

Stein, M., M. Smith, M. Henningsen y E. Silver, Implementing Standards-Based Mathematics Instruction: A Casebook for Professional Development, $2^{a}$ Ed., VA: NCTM, New York, USA (2009)

Stanley, D. y M. Sundström, Extended analyses: finding deep structure in standard high school mathematics, doi: https://doi.org/10.1007/s10857-007-9050-z, Journal Mathematics Teacher Education, 10, 391-397 (2007)

SEP, Programas de Estudio 2011 Guía para el Maestro, Educación Básica Matemáticas, SEP, México (2011)

Thomas, M., I. Druck y otros cinco autores, Survey team 4: key mathematical concepts in the transition from secondary to university; In 12th International Congress on Mathematical Education, 90-136, Seoul, Korea, 8-15 July (2012)

Tirado, F., E. Backhoff, N. Larrazolo y M. Rosas, Validez predictiva del Examen de Habilidades y Conocimientos Básicos (EXHCOBA), ISSN: 1405-6666, Revista Mexicana de Investigación Educativa, 11 (3), 67-84 (1997)

Varsavsky, C., Chances of success in and engagement with mathematics for students who enter university with a weak mathematics background, doi: 10.1080/0020739X.2010.493238, International Journal of Mathematical Education in Science and Technology, 41(8), 1037-1049 (2010) 
